\title{
Sestrin2 Attenuates Cellular Senescence by Inhibiting NADPH Oxidase 4 Expression
}

\author{
Chae Young Hwang ${ }^{1,{ }^{*}}$, Ying-Hao Han ${ }^{1,}$, Seung-Min Lee ${ }^{1}$, Sang-Mi Cho ${ }^{1}$, Dae-Yeul Yu ${ }^{1,2}$, Ki-Sun Kwon ${ }^{1,3}$ \\ ${ }^{1}$ Aging Research Center, Korea Research Institute of Bioscience and Biotechnology, Daejeon, Korea \\ ${ }^{2}$ GHBIO Inc., Daejeon, Korea \\ ${ }^{3}$ Aventi Inc., Daejeon, Korea
}

Corresponding Author:

Ki-Sun Kwon, $\mathrm{PhD}$

Aging Research Center, Korea Research

Institute of Bioscience and

Biotechnology, 125 Gwahak-ro,

Yuseong-gu, Daejeon 34141, Korea

ORCID:

https://orcid.org/0000-0002-6401-2735

Dae-Yeul Yu, PhD

Aging Research Center, Korea Research Institute of Bioscience and

Biotechnology, 125 Gwahak-ro,

Yuseong-gu, Daejeon 34141, Korea

E-mail: dyyu10@kribb.re.kr

${ }^{*}$ These authors contributed equally to this work.

Current affiliation: Chae Young Hwang

(Laboratory for Systems Biology and

Bio-inspired Engineering, Department

of Bio and Brain Engineering, Korea

Advanced Institute of Science and

Technology, Daejeon, Korea), Ying-

Hao Han (College of Life Science and

Technology, Heilongjiang Bayi

Agricultural University, Daqing,

China).

Received: August 8, 2020

Revised: October 14, 2020

Accepted: October 21, 2020
Background: Sestrin2 (Sesn2) is involved in the maintenance of metabolic homeostasis and aging via modulation of the $5^{\prime}$ AMP-activated protein kinase-mammalian target of rapamycin (AMPK-mTOR) pathway. Methods: Wild-type and Sesn2 knockout (KO) mice of the 129/SvJ background were maintained in a pathogen-free authorized facility under a 12-hour dark/light cycle at $20^{\circ} \mathrm{C}-22^{\circ} \mathrm{C}$ and $50 \%-60 \%$ humidity. Mouse embryonic fibroblasts (MEFs) were prepared from 13.5-day-old embryos derived from Sesn2-KO mice mated with each other. Results: The MEFs from Sesn2-KO mice showed enlarged and flattened morphologies and senescence-associated $\beta$-galactosidase activity, accompanied by an elevated level of reactive oxygen species. These senescence phenotypes recovered following treatment with $\mathrm{N}$-acetyl-cysteine. Notably, the mRNA levels of NADPH oxidase 4 (NOX4) and transforming growth factor (TGF)- $\beta$ were markedly increased in Sesn2-KO MEFs. Treatment of Sesn2-KO MEFs with the NOX inhibitor diphenyleneiodonium and the TGF- $\beta$ inhibitor SB431542 restored cell growth inhibited by Sesn2-KO. Conclusion: Sesn 2 attenuates cellular senescence via suppression of TGF- $\beta$ - and NOX4-induced reactive oxygen species generation and subsequent inhibition of AMPK.

Key Words: NOX4, Reactive oxygen species, Senescence, Sestrin2

\section{INTRODUCTION}

In mammals, the Sestrin (Sesn) family of stress-sensitive genes comprises three members: Sesn 1, Sesn2, and Sesn $3 .{ }^{1)}$ Sesns have dual biochemical functions: first, they act as antioxidants that control the activity of peroxiredoxins (PRXs), ${ }^{2)}$ in which the expres- 
sion level of Sesn 2 is critical for redox homeostasis. The ectopic expression of Sesn 2 significantly reduces reactive oxygen species (ROS) levels and increases cell viability, whereas the suppression of Sesn2 increases intracellular ROS levels and reduces cell viability following hydrogen peroxide exposure. ${ }^{2)}$ Sesns also function as inhibitors of target of rapamycin complex 1 (TORC1) signaling. ${ }^{3)}$ Drosophila Sesn (dSesn) has been reported to prevent age-associated pathologies, including fat accumulation and cardiac and skeletal muscle degeneration, by promoting a feedback loop that prevents excessive TORC1 activation and ROS accumulation. ${ }^{3)}$ As ROS accumulation $^{4)}$ and TORC1 activation ${ }^{5,6)}$ are associated with accelerated aging and the development of age-associated pathologies in both invertebrates and vertebrates, we tested whether the loss of Sesn 2 induces cellular senescence in mouse embryonic fibroblasts (MEFs) before investigating Sesn 2 knockout (KO) mice at the organismal level.

Cellular senescence is an irreversible growth arrest state provoked by diverse stresses. ${ }^{4)}$ In particular, the relationship between cellular senescence and ROS was confirmed by the observation that treatment with exogenous hydrogen peroxide triggers certain primary cells to quickly enter senescence. ${ }^{7-9)}$ The results of the present study showed that Sesn2 KO induced cellular senescence in MEFs and demonstrated that Sesn2 was an important regulator of cellular senescence via NADPH oxidase 4 (NOX4)-dependent ROS generation and subsequent activation of AMP-activated protein kinase (AMPK).

\section{MATERIALS AND METHODS}

\section{Mice}

Sesn2-KO mice were produced by transferring two-cell embryos deficient in Sesn2 into the oviduct of a foster mother. The Sesn2KO embryos were provided by the Mutant Mouse Resource \& Research Centers at the University of California, Davis. Wild-type (WT) and Sesn2-KO mice with the 129/SvJ background were maintained in a pathogen-free authorized facility at the Korea Research Institute of Bioscience and Biotechnology (KRIBB) under a 12 -hour dark/light cycle at $20^{\circ} \mathrm{C}-22^{\circ} \mathrm{C}$ and $50 \%-60 \%$ humidity. All animal procedures were conducted according to the guidelines of the Institutional Animal Care and Use Committee of KRIBB.

\section{Preparation of MEFs}

MEFs were prepared from 13.5-day-old embryos derived from Sesn2-KO mice mated with each other. The head, tail, and viscera were removed, and the remaining body was minced, dispersed in $0.25 \%$ trypsin/EDTA, and incubated in $5 \% \mathrm{CO}_{2}$ at $37^{\circ} \mathrm{C}$ for 30 minutes. Large fragments were removed, and the cell suspensions were plated on $10-\mathrm{cm}$ plates and incubated at $37^{\circ} \mathrm{C}$ until confluent.

\section{Cell Culture}

WT and Sesn2 KO MEFs, as well as $293 \mathrm{~T}$ cells, were cultured in Dulbecco's Modified Eagle's Medium supplemented with $10 \%$ fetal bovine serum, $20 \mathrm{mM}$ HEPES, and antibiotics (Life Technologies Corp., Carlsbad, CA, USA) at $37^{\circ} \mathrm{C}$ in a humidified atmosphere containing $5 \% \mathrm{CO}_{2}$.

\section{Immunoblotting}

Immunoblotting was performed as described previously." Briefly, cells were lysed in lysis buffer (20 mM HEPES, pH 7.2, $50 \mathrm{mM}$ $\mathrm{NaCl}, 0.5 \%$ Triton X-100, 10\% glycerol, $1 \mu \mathrm{g} / \mathrm{mL}$ aprotinin, $1 \mu \mathrm{g}$ leupeptin, $1 \mathrm{mM} \mathrm{Na}_{3} \mathrm{VO}_{4}$, and $1 \mathrm{mM} \mathrm{NaF}$ ). Antibodies against the

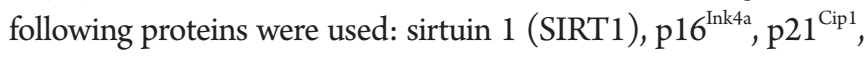
$\alpha$-actinin, human influenza hemagglutinin (HA), and $\beta$-actin (all from Santa Cruz Biotechnology, Santa Cruz, CA, USA); phospho-Smad3, phospho-AMPK, phospho-Akt, p53, and AMPK (all from Cell Signaling Technology Inc., Danvers, MA, USA); Sesn2 (ProteinTech Group Inc., Chicago, IL, USA); nucleoredoxin (R\&D Systems Inc., Minneapolis, MN, USA); $a$-tubulin (Calbiochem, San Diego, CA, USA); $\gamma$-tubulin (Abcam Inc., Cambridge, MA, USA); paired related homeobox 1 (PRX1), PRX2, and PRX3; anti-thioredoxin (TRX; a kind gift from Dr. Ho Zoon Chae, Chonnam National University, Gwangju, Korea); and anti-malate dehydrogenase 1 (MDH1; a kind gift from Dr. HongDuk Youn, Seoul National University, Seoul, Korea).

\section{Reverse-Transcription Polymerase Chain Reaction (RT-PCR)}

Total RNA was isolated using RNA-spin (Intron Biotechnology Inc., Seongnam, Korea). cDNA was synthesized from $1 \mu \mathrm{g}$ of total RNA using a DiaStar RT Kit (SolGent, Gwanpyeong-dong, Korea). The primers are listed in Table 1 . The housekeeping genes $\beta$-actin and GAPDH were used as endogenous controls for normalization.

\section{Determination of ROS Production}

The $2^{\prime}, 7^{\prime}$-dichlorofluorescein diacetate (DCFH-DA; Life Technologies Corp.) oxidation-sensitive probe was used to measure intracellular ROS levels by flow cytometry. Briefly, cells were incubated with $10 \mu \mathrm{M}$ DCFH-DA for 30 minutes. For flow cytometry analysis, the cells were detached by trypsinization, washed once in phosphate-buffered saline (PBS), and resuspended in $800 \mu \mathrm{L}$ PBS. Flow cytometric analyses (10,000 events per sample) were performed using a FACSCalibur system (BD Biosciences, San Jose, CA, USA) with excitation and emission wavelengths of $485 \mathrm{~nm}$ 
Table 1. Primer sequences

\begin{tabular}{|c|c|c|}
\hline Genes & Forward primer $\left(5^{\prime}-3^{\prime}\right)$ & Reverse primer $\left(5^{\prime}-3^{\prime}\right)$ \\
\hline \multicolumn{3}{|c|}{ Mouse primers } \\
\hline Sesn2 & ATG ATC GTA GCG GAC TCC GAG & CAA GGC CTG GAT ATG CTC CTT \\
\hline NOX2 & ССС АСС СТТ ТСА АAА ССА ТТ & ACT TGG ATA CCT TGG GGC AC \\
\hline NOX3 & GAA GGC TTT CTA TGG GCG AC & ATC CAC AGA AGA ACA CGC CA \\
\hline NOX4 & CCT GGA AGA ACC CAA GTT CC & CGG TAA AGT CTC TCC GCA CA \\
\hline TRX & ATG GTG AAG CTG ATC GAG AGC & TTA GGC ATA TTC AGT AAT AGA \\
\hline PRX1 & ATG TCT TCA GGAAAT GCAAAA & CTT GAT GGT ATC ACT GCC AGG \\
\hline $\mathrm{PRX} 2$ & ATG GCC TCC GGC AAC GCG CAA & CTT GAT GGT GTC ACT GCC GGG \\
\hline NRX & ATG TCG GGC TTC CTG GAG GAG & CGC AAT AAC TTC CCG GAA GGG \\
\hline TGF- $\beta$ & GCT GCG GCT ACT GCC GCT TCT GCT & CGC CAG GAA TTG TTG CTA TAT TTC \\
\hline Sesn2 & GAG CTC ATT CAG GCT CTG GTC CTG & CAG CAG GCT CTC TGA CTT CTC CAG \\
\hline NOX4 & GAT GAC TGG AAA CCA TAC AAG & TAAAAG TTT CCA CCG AGG ACG \\
\hline TGF- $\beta$ & TGG CTA CTG GTG CTG ACG CCT & TTC TCG GAG CTC TGA TGT GTT GA \\
\hline GAPDH & GAG TCA ACG GAT TTG GTC GT & TTG ATT TTG GAG GGA TCT CG \\
\hline
\end{tabular}

and $538 \mathrm{~nm}$, respectively, and evaluated using CellQuest software.

\section{SA- $\beta$-Gal Activity}

Senescence-associated $\beta$-galactosidase (SA- $\beta$-Gal) staining was performed as previously described. ${ }^{10)}$ Briefly, the cells were washed in PBS, fixed in $2 \%$ formaldehyde and $0.2 \%$ glutaraldehyde for 5 minutes, and washed three times. Next, the cells were incubated in fresh SA- $\beta$-Gal stain solution-1 mg/mL X-gal (5-bromo-4chloro-3-indolyl b-D-galactoside) stock $(20 \mathrm{mg} / \mathrm{mL}$ in dimethylformamide)/40 mM citric acid/sodium phosphate, $\mathrm{pH} 6.0 / 5 \mathrm{mM}$ potassium ferrocyanide $/ 5 \mathrm{mM}$ potassium ferricyanide/ $150 \mathrm{mM}$ $\mathrm{NaCl}_{2}$-for $12-16$ hours at $37^{\circ} \mathrm{C}$. Blue cells were counted under an inverted microscope (Axiovert25; Carl Zeiss, Oberkochen, Germany).

\section{Cell Proliferation Assay}

Relative cell proliferation was measured as previously described. ${ }^{9}$ Briefly, WST-1 solution (DoGen, Seoul, Korea) was added to the cells for 2 hours, and the absorbance was measured at $450 \mathrm{~nm}$ using a VICTOR3 Multilabel Plate Reader (PerkinElmer Inc., Waltham, MA, USA). The cells were then treated with the TGF- $\beta$ type I receptor kinase inhibitor SB431542 (SB; $5 \mu \mathrm{M}$, Calbiochem), the NOX inhibitor diphenyleneiodonium (DPI; $5 \mu \mathrm{M}$, Calbiochem), and the AMPK inhibitor compound C ( $5 \mu \mathrm{M}$, Sigma-Aldrich, St. Louis, MO, USA) for 48 hours after plating.

\section{Transfection}

The 293T cells were transfected with the HA-mCherry human Sesn2 (hSesn2) and SBE4 reporter plasmid, pretreated with TGF- $\beta$ ( $2 \mathrm{ng} / \mathrm{mL})$ for 24 hours, and subjected to a reporter assay. Relative luciferase activity was analyzed using a Promega Luciferase Assay System (Promega Corp., Madison, WI, USA) according to the manufacturer's instructions.

\section{Statistical Analysis}

All data are presented as mean \pm SEM (standard error of the mean) of triplicate independent experiments. Statistical analysis was performed using a two-tailed unpaired Student t-tests. Statistical analyses were performed using Microsoft Excel 2016.

\section{RESULTS}

\section{Loss of Sesn2 Induces Cellular Senescence}

To determine the role of Sesn2 in cellular senescence, we examined the effect of Sesn2-KO on senescence in MEFs. MEFs isolated from Sesn2-KO mice exhibited obvious cellular senescence phenotypes (Fig. 1A). We observed SA- $\beta$-Gal-positive cells among Sesn2-KO MEFs but not among WT MEFs (Fig. 1A, 1B). The Sesn2-KO MEFs also became flattened and enlarged (Fig. 1A), constituting the morphological changes characterizing senescent cells. Next, we examined whether Sesn2-KO prevented cell proliferation. Compared with WT MEFs, Sesn2-KO MEFs showed a 


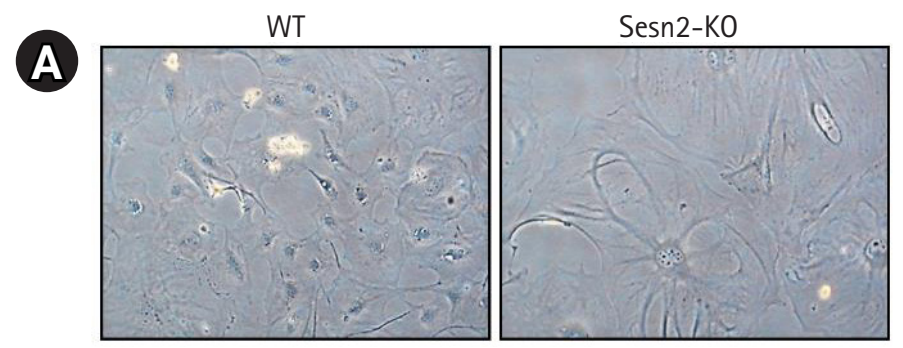

Phase contrast

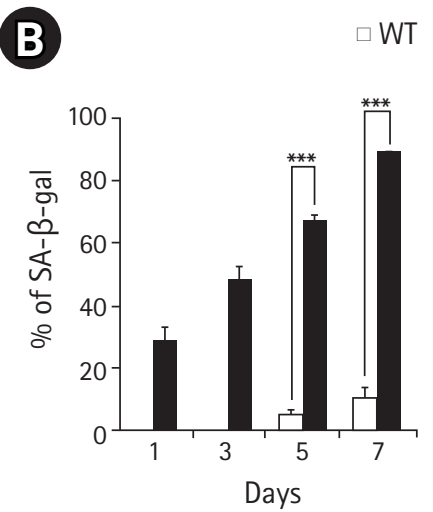

WT - Sesn2-KO

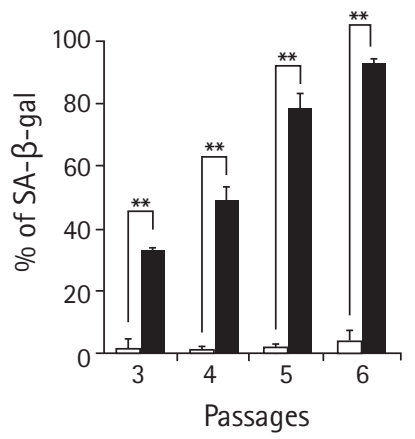

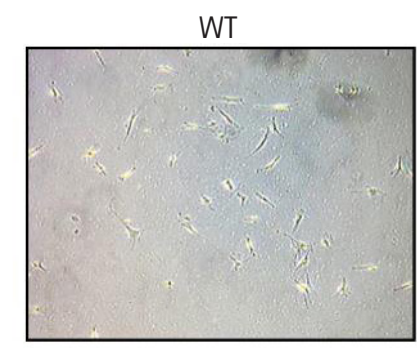

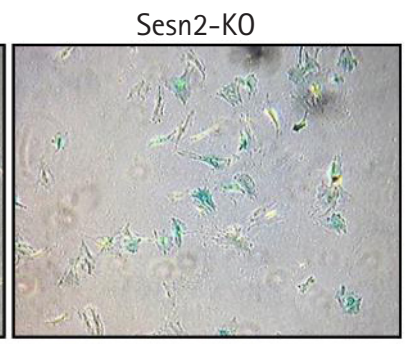

SA- $\beta$-gal

Fig. 1. Loss of Sesn2 induces cellular senescence in mouse embryonic fibroblasts (MEFs). (A) Representative phase microscopy images of wildtype (WT) and Sesn 2 knockout (KO) MEFs stained with X-gal. Left, phase contrast; right, SA- $\beta$-Gal activity ( $\mathrm{n}=3$ independent experiments). (B) Percentage of SA- $\beta$-Gal-positive cells. MEFs (passage 3) were cultured in 6-well plates for 24 hours, and SA- $\beta$-Gal-positive cells were counted. Left, 1-7 days; right, passages 3-6 ( $\mathrm{n}=3$ independent experiments). (C) Cell proliferation was assessed using WST-1 assays (MEFs, passage 5). (D) Immunoblotting results using anti-Sesn2, anti-p53, anti-p16Ink4a, and anti-p21Cip1 antibodies in cell lysates from WT and Sesn2-KO MEFs. $\beta$-actin was used as a loading control (passage 5). The data represent as mean \pm SEM (standard error of the mean). Two-tailed unpaired Student t-tests were used for statistical analysis. ${ }^{* *} \mathrm{p}<0.01,{ }^{* * *} \mathrm{p}<0.001$.

$25 \%$ decrease in proliferation (Fig. 1C). These results suggested that Sesn 2 was involved in the progression of cellular senescence. However, despite increased cellular senescence, there were no obvious differences in the basal levels of $\mathrm{p} 16^{\mathrm{Ink4a}}$ and $\mathrm{p} 21^{\mathrm{Cip} 1}$, which induce cell cycle arrest and accelerated senescence, ${ }^{11)}$ in Sesn2-KO MEFs compared to WT MEFs (Fig. 1D).

\section{Loss of Sesn2 Generates ROS}

The characteristics of Sesn deficiency have been reported previously in flies. ${ }^{3)}$ To investigate the mechanisms by which the loss of Sesn2 induces cellular senescence in MEFs, we measured intracellular ROS levels in Sesn2-KO MEFs by staining with DCFH-DA, a peroxide-sensitive fluorescent probe. DCFH-DA passively enters the cell, where it reacts with ROS to form the highly fluorescent compound dichlorofluorescein. As shown in Fig. 2A, the fluorescence intensity of dichlorofluorescein was significantly increased in Sesn2-KO MEFs compared to that in WT MEFs on days 3, 5, and 7 after plating. To explore the effect of ROS on cellular senescence, we analyzed whether changes in ROS levels affected cellular senescence in Sesn2-KO MEFs. Treatment with the antioxidant $\mathrm{N}$-acetyl-cysteine (NAC) significantly decreased the SA- $\beta$-Gal activity in
Sesn2-KO MEFs (Fig. 2B, 2C). These results suggested that the loss of Sesn 2 accelerated cellular senescence via ROS generation.

\section{Loss of Sesn 2 Triggers NOX4}

An increase in ROS levels by lowering antioxidant levels reportedly accelerates cellular senescence, whereas an increase in ROS scavenging delays senescence. ${ }^{12)}$ Therefore, we measured the levels of antioxidant proteins in Sesn2-KO MEFs to determine whether the loss of Sesn 2 increases ROS levels by regulating the expression of antioxidant enzymes. No obvious changes were observed in the mRNA expression levels and protein abundance of several antioxidants, including PRX1, PRX2, PRX3, and TRX, between WT and Sesn2-KO MEFs (Fig. 3A, 3B), suggesting that cellular senescence induced by loss of Sesn 2 was not mediated by a decrease in antioxidant levels. However, NRX protein levels were slightly increased in Sesn2-KO MEFs (Fig. 3B).

As NOX family members generate $\mathrm{ROS},{ }^{13)}$ we investigated which NOX family members were involved in Sesn 2 signaling. We analyzed the expression patterns of DUOX1, NOX1, NOX2, NOX3, and NOX4 in WT and Sesn2-KO MEFs by RT-PCR. We observed markedly increased NOX4 expression in Sesn2-KO 

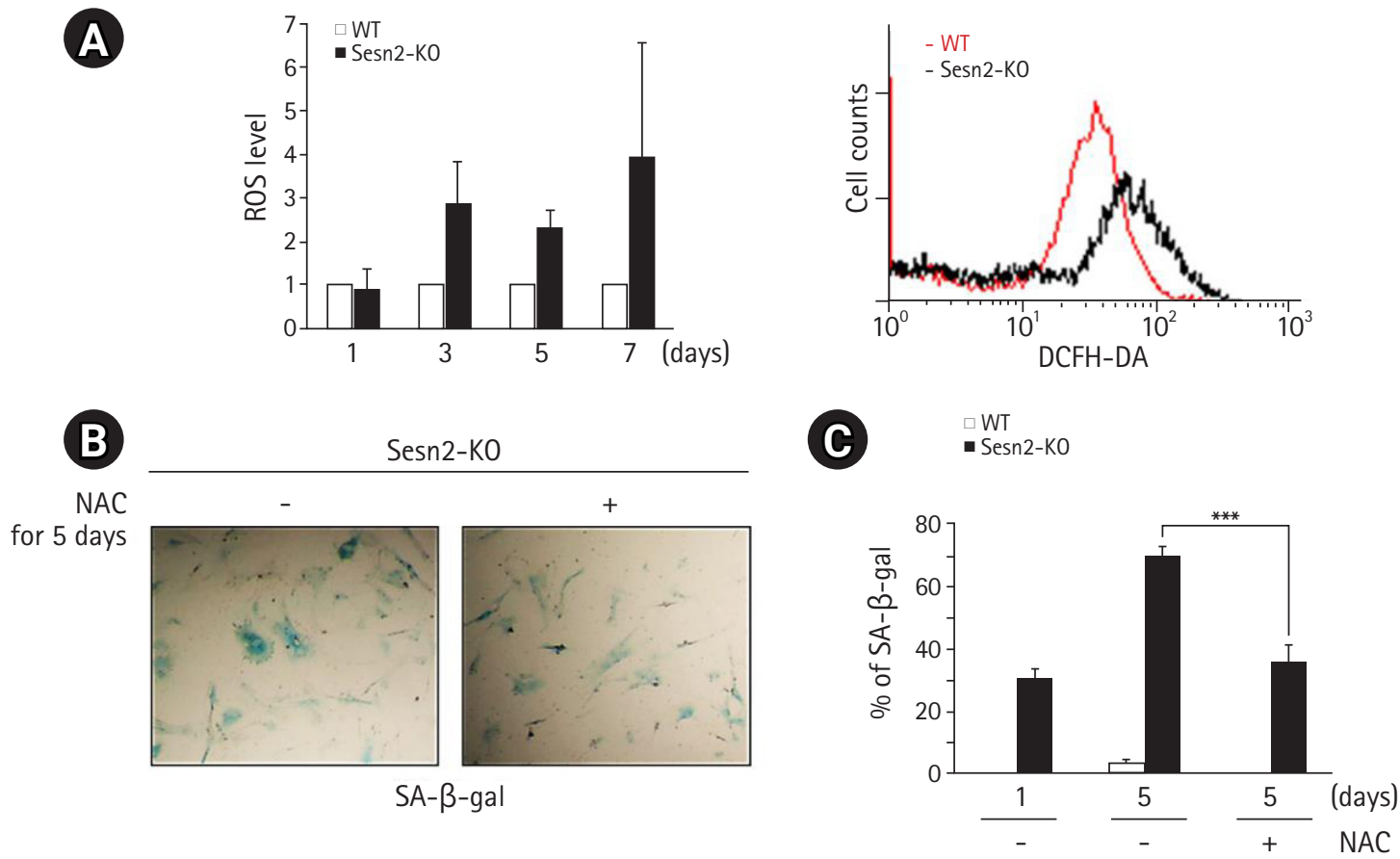

Fig. 2. Loss of Sesn 2 causes increased reactive oxygen species (ROS) production in mouse embryonic fibroblasts (MEFs). (A) Relative ROS levels. MEFs (passage 5) were harvested at 1-7 days after plating ( $\mathrm{n}=3$ independent experiments, left), and relative ROS levels were measured by fluorescence-activated cell sorting (FACS) using $2^{\prime}, 7^{\prime}$-dichlorofluorescin diacetate (DCFH-DA, right). The red and black curves indicate wildtype (WT) and Sesn2 knockout (KO) MEFs, respectively. (B) Representative phase microscopy images of Sesn2-KO MEFs stained with X-gal to show SA- $\beta$-Gal activity. Sesn2-KO MEFs (passage 3) were treated with N-acetyl-cysteine (NAC). After 5 days, SA- $\beta$-Gal-positive cells were measured by X-gal staining. (C) Percentage of SA- $\beta$-Gal-positive cells. The data represent as mean \pm SEM (standard error of the mean). Twotailed unpaired Student t-tests were used for statistical analysis. ${ }^{* *} \mathrm{p}<0.001$.

MEFs (Fig. 3C), suggesting that NOX4 played an important role in ROS production in Sesn2-KO MEFs.

Because TGF- $\beta$ upregulates NOX4, ${ }^{14,15)}$ we measured the mRNA levels of TGF- $\beta$ to determine whether the loss of Sesn 2 increased TGF- $\beta$ levels. We observed increased mRNA levels of TGF- $\beta$ in Sesn2-KO MEFs (Fig. 3C). Next, we induced the overexpression of hSesn 2 in $293 \mathrm{~T}$ cells. We found that hSesn 2 expression markedly decreased TGF- $\beta$ promoter activity (Fig. 3D), Smad3 phosphorylation (Fig. 3E), and mRNA levels of TGF- $\beta$, ultimately leading to a decrease in NOX4 mRNA levels (Fig. 3F). Together, these results indicated that Sesn 2 is a key regulator of the TGF- $\beta$-mediated NOX4 pathway.

\section{AMPK Activation is Involved in the Loss of Sesn2-Induced}

\section{Senescence}

Abundant evidence indicates that a rise in intracellular ROS levels contributes to cellular senescence. ${ }^{4,12)}$ Moreover, ROS also induces ATP depletion. ${ }^{16)}$ AMPK is an energy sensor that is activated by increased levels of intracellular AMP. Generally, AMPK activation turns on catabolic pathways that generate ATP while also inhibiting cell proliferation and biosynthetic processes that consume
ATP. Thus, we hypothesized that elevated ROS levels lead to AMPK activation in Sesn2-KO MEFs, likely via a decline in ATP levels. We found that the loss of Sesn2 led to AMPK activation in MEFs with a reduction in Akt phosphorylation and SIRT1 protein levels (Fig. 4A). As MDH1 knockdown induces senescence in human fibroblasts, ${ }^{17)}$ we examined whether $\mathrm{MDH} 1$ was related to senescence in Sesn2-KO MEFs. No changes were observed in the protein levels of MDH1 in Sesn2-KO MEFs (Fig. 4A). To clarify the effect of ROS on AMPK activation, we treated Sesn2-KO MEFs with NAC and observed significantly suppressed AMPK activation (Fig. 4B). We then investigated the proliferation of Sesn2-KO MEFs using the TGF- $\beta$ type I receptor kinase inhibitor $\mathrm{SB}$, the NOX inhibitor DPI, and the AMPK inhibitor compound C. $\mathrm{SB}, \mathrm{DPI}$, and compound $\mathrm{C}$ treatment significantly increased the growth of Sesn2-KO MEFs compared to that of WT MEFs (Fig. 4C), indicating that the TGF- $\beta / \mathrm{NOX} 4 / \mathrm{AMPK}$ pathway may contribute to proliferation defects in Sesn2-KO MEFs (Fig. 4D).

\section{DISCUSSION}

The results of this study allowed us to characterize Sesn2 as a cru- 

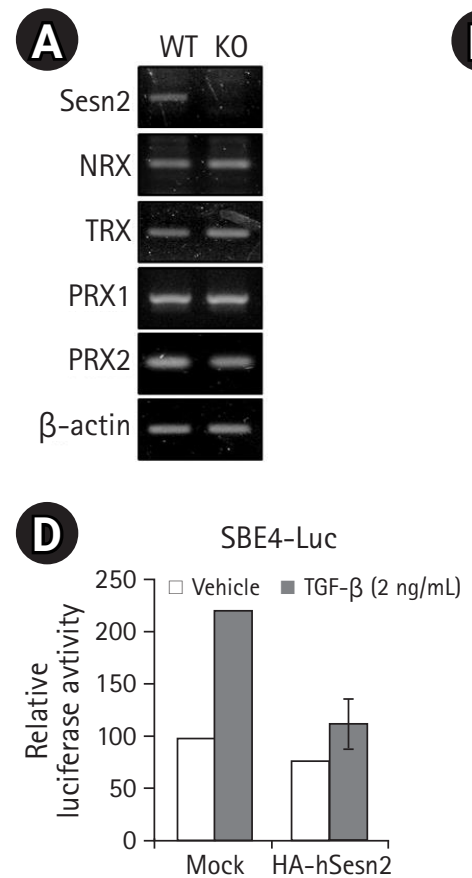

B

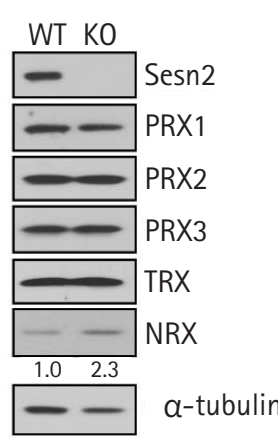

E

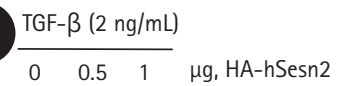

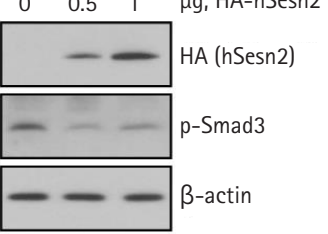

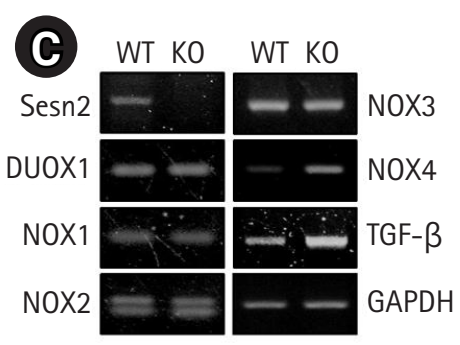

$\boldsymbol{F}$

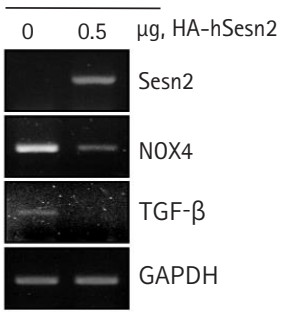

Fig. 3. Loss of Sesn2 results in NOX4 accumulation. (A) mRNA levels of Sesn2, NRX, TRX, PRX1, and PRX2 in wild-type (WT) and Sesn2 knockout (KO) mouse embryonic fibroblasts (MEFs) (passage 5). $\beta$-actin was used as a loading control. (B) Immunoblotting results using the indicated antibodies in cell lysates from WT and Sesn2-KO MEFs (passage 5). $\alpha$-tubulin was used as a loading control. The protein levels of NRX were normalized to those of $\gamma$-tubulin and quantified using ImageJ software. (C) mRNA levels of Sesn2, DUOX1, NOX1, NOX2, NOX3, NOX4, TGF- $\beta$, and GAPDH in WT and Sesn2-KO MEFs (passage 5). (D) Relative luciferase activity. The 293T cells were transfected with an HA-mCherry Sesn 2 and SBE4 reporter plasmid, treated with TGF- $\beta(2 \mathrm{ng} / \mathrm{mL})$ for 24 hours, and subjected to a reporter assay. (E) Immunoblotting results using anti-HA (HA-mCherry Sesn2), anti-phospho-Smad3, and anti- $\beta$-actin antibodies. (F) Sesn2, NOX4, TGF- $\beta$, and GAPDH mRNA expression in hSesn2-overexpressing $293 \mathrm{~T}$ cells. The data represent as mean \pm SEM (standard error of the mean). Two-tailed unpaired Student t-tests were used for statistical analysis. ${ }^{* *} \mathrm{p}<0.01$.
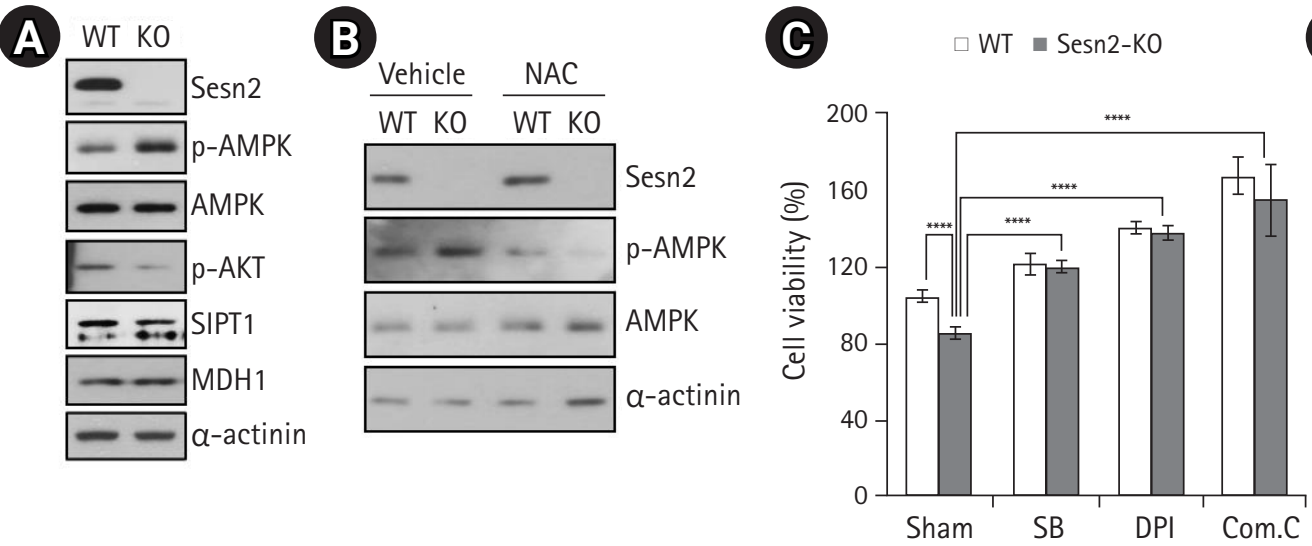

D

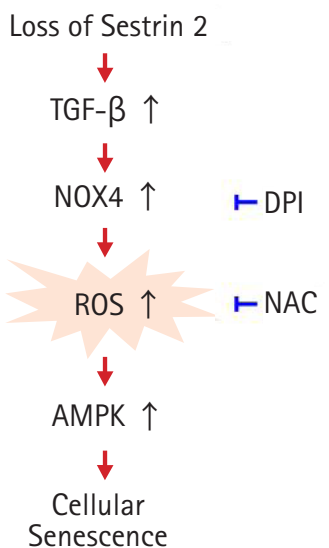

Fig. 4. Loss of Sesn2 triggers AMPK (AMP-activated protein kinase) activation. (A) Immunoblotting results using the indicated antibodies in cell lysates from wild-type (WT) and Sesn 2 knockout (KO) mouse embryonic fibroblasts (MEFs) (passage 5). (B) Decreased AMPK activation by $\mathrm{N}$-acetyl-cysteine (NAC) application in Sesn2-KO MEFs (passage 5). Immunoblotting results using anti-Sesn2, anti-phospho-AMPK, antiAMPK, and anti- $\alpha$-actinin antibodies. (C) Cell viability was assessed using the WST-1 assay ( $\mathrm{n}=3$ independent experiments). (D) Schematic representation of the proposed pathway for the regulation of cellular senescence by Sesn2. The data represent as mean \pm SEM (standard error of the mean). Two-tailed unpaired Student t-tests were used for statistical analysis. ${ }^{* * *} \mathrm{p}<0.0001$. 
cial regulator of cellular senescence. Sesn 2 was first reported as a disulfide reductase of $\mathrm{PRX},{ }^{2)}$ and $\mathrm{dSesn}$ has been shown to prevent excessive ROS accumulation. ${ }^{3)}$ In contrast, one study reported that Sesn2 does not function as a reductase of cysteine sulfinic acid in PRXs. ${ }^{18)}$

We observed that Sesn2-KO MEFs generated ROS. Loss of Sesn2 has been associated with the induction of TGF- $\beta$ signaling independent of ROS accumulation. ${ }^{19)}$ TGF- $\beta$ has also been shown to upregulate NOX4, which produces ROS. ${ }^{14,15)}$ NOX4 overexpression also induces cellular senescence. ${ }^{20)}$ On the basis of these reports, we evaluated whether TGF- $\beta$ and NOX4 were involved in ROS generation in association with the senescence phenotype in Sesn2-KO MEFs. RT-PCR analysis revealed that NOX4 is the major NADPH oxidase isoform expressed in Sesn2-KO MEFs (Fig. 3C), suggesting that the loss of Sesn 2 induces TGF- $\beta$-mediated NOX4 expression.

Cellular senescence participates in four complex biological processes (tumor suppression, tumor promotion, aging, and tissue repair), some of which have opposing effects. ${ }^{21)}$ Furthermore, cellular senescence is triggered by a complex signaling network involving the interaction of multiple proteins, including those associated with mitochondrial function, ROS, senescence, and chromatin remodeling. ${ }^{17,22)}$

Lowered ATP production and subsequently an elevated AMP:ATP ratio and increased AMPK activity are associated with age. ${ }^{23)}$ Moreover, ROS can activate AMPK via decreased ATP levels. ${ }^{24}$ Evidence suggests that AMPK plays a role in cellular senescence. Activated AMPK leads to phosphorylation of p53 at serine 15, which induces p53-dependent cellular senescence. ${ }^{25)}$ Activated AMPK has also been shown to inhibit the RNA-binding protein $\mathrm{HuR}^{23)} \mathrm{HuR}$ levels are reduced in senescent cells and HuR overexpression restores a young phenotype, whereas a reduction in HuR expression accentuates senescence-associated morphology. ${ }^{26)}$ Finally, activated AMPK contributes to the inhibition of mammalian target of rapamycin (mTOR) signaling by activation of the tuberous sclerosis complex 2 (TSC2) in response to energy stress. ${ }^{27}$ ) Activation of TSC2 by AMPK strongly suppresses cell proliferation. ${ }^{28)}$ In addition, AMPK directly phosphorylates mTOR at threonine 2446 following stimulation with insulin, thereby inhibiting mTOR action. ${ }^{29)}$ Thus, AMPK can inhibit mTOR activity to limit cell proliferation as well as protein synthesis both directly and indirectly (via TSC2).

Both mRNA and protein levels of SIRT 1 are markedly decreased during senescence; a decrease in SIRT1 levels leads to the acetylation of liver kinase B1 (LKB1), which, in turn, induces AMPK-dependent senescence. Moreover, the phosphorylation of Akt at serine 473 is markedly decreased in senescent cells. The inhibition of Akt induces cellular senescence, and liver kinase B1 overexpression markedly decreases Akt activity when endogenous SIRT1 is inhibited. ${ }^{30)}$ Although we did not elucidate the detailed signaling cascade downstream of AMPK, we observed slightly decreased SIRT1 protein levels and significantly inhibited phosphorylation of Akt at serine 473 in Sesn2-KO MEFs compared to those in WT MEFs (Fig. 4A). Our results showed that Sesn2 was a key factor of senescence via AMPK activation. This role of Sesn2 is likely mediated by a NOX4-mediated increase in ROS levels, as NAC prevented the increase in ROS levels and attenuated AMPK activation (Fig. 4B). We also found that inhibitor-mediated abrogation of TGF- $\beta$ and NOX4 activities resulted in increased cell proliferation (Fig. 4C). Our results suggest a novel Sesn2-mediated pathway that may underlie the induction of NOX4, generation of ROS, and subsequent activation of AMPK in cellular senescence.

\section{ACKNOWLEDGMENTS}

\section{CONFLICT OF INTEREST}

The researchers claim no conflicts of interest.

\section{FUNDING}

This study was supported by grants from the Bio \& Medical Technology Development Program (No. 20110030133 and 2017M3A9D8048708 to Ki-Sun Kwon) of the National Research Foundation (NRF) funded by the Korean government (Ministry of Science and ICT) and the KRIBB Research Initiative Program.

\section{AUTHOR CONTRIBUTIONS}

Conceptualization: $\mathrm{CYH}, \mathrm{YHH}$; Investigation, SML, SMC; Funding acquisition, KSK; Writing-original draft: CYH, YHH, DYY, KSK. Supervision: KSK.

\section{REFERENCES}

1. Budanov AV, Shoshani T, Faerman A, Zelin E, Kamer I, Kalinski $\mathrm{H}$, et al. Identification of a novel stress-responsive gene Hi95 involved in regulation of cell viability. Oncogene 2002;21:601731.

2. Budanov AV, Sablina AA, Feinstein E, Koonin EV, Chumakov PM. Regeneration of peroxiredoxins by p53-regulated sestrins, homologs of bacterial AhpD. Science 2004;304:596-600.

3. Lee JH, Budanov AV, Park EJ, Birse R, Kim TE, Perkins GA, et al. Sestrin as a feedback inhibitor of TOR that prevents age-related pathologies. Science 2010;327:1223-8.

4. Balaban RS, Nemoto S, Finkel T. Mitochondria, oxidants, and aging. Cell 2005;120:483-95.

5. Passtoors WM, Beekman M, Deelen J, van der Breggen R, Maier 
AB, Guigas B, et al. Gene expression analysis of mTOR pathway: association with human longevity. Aging Cell 2013;12:24-1.

6. Zhang Y, Lanjuin A, Chowdhury SR, Mistry M, Silva-Garcia CG, Weir HJ, et al. Neuronal TORC1 modulates longevity via AMPK and cell nonautonomous regulation of mitochondrial dynamics in C. elegans. Elife 2019;8:e49158.

7. Chen QM, Bartholomew JC, Campisi J, Acosta M, Reagan JD, Ames BN. Molecular analysis of H2O2-induced senescent-like growth arrest in normal human fibroblasts: p53 and Rb control G1 arrest but not cell replication. Biochem J 1998;332(Pt 1):4350.

8. Frippiat C, Chen QM, Remacle J, Toussaint O. Cell cycle regulation in $\mathrm{H}(2) \mathrm{O}(2)$-induced premature senescence of human diploid fibroblasts and regulatory control exerted by the papilloma virus E6 and E7 proteins. Exp Gerontol 2000;35:733-45.

9. Hwang CY, Lee SM, Park SS, Kwon KS. CDK2 differentially controls normal cell senescence and cancer cell proliferation upon exposure to reactive oxygen species. Biochem Biophys Res Commun 2012;425:94-9.

10. Dimri GP, Lee X, Basile G, Acosta M, Scott G, Roskelley C, et al. A biomarker that identifies senescent human cells in culture and in aging skin in vivo. Proc Natl Acad Sci U S A 1995;92:9363-7.

11. Mirzayans R, Andrais B, Scott A, Paterson MC, Murray D. Single-cell analysis of p16(INK4a) and p21(WAF1) expression suggests distinct mechanisms of senescence in normal human and Li-Fraumeni Syndrome fibroblasts. J Cell Physiol 2010;223: 57-67.

12. Lu T, Finkel T. Free radicals and senescence. Exp Cell Res 2008;314:1918-22.

13. Bedard K, Krause KH. The NOX family of ROS-generating NADPH oxidases: physiology and pathophysiology. Physiol Rev 2007;87:245-313.

14. Carmona-Cuenca I, Roncero C, Sancho P, Caja L, Fausto N, Fernandez $\mathrm{M}$, et al. Upregulation of the NADPH oxidase NOX4 by TGF-beta in hepatocytes is required for its pro-apoptotic activity. J Hepatol 2008;49:965-76.

15. Tong X, Hou X, Jourd'heuil D, Weisbrod RM, Cohen RA. Upregulation of Nox4 by TGF $\{$ beta 1 oxidizes SERCA and inhibits NO in arterial smooth muscle of the prediabetic Zucker rat. Circ Res 2010; 107:975-83.

16. Villena J, Henriquez M, Torres V, Moraga F, Diaz-Elizondo J, Arredondo C, et al. Ceramide-induced formation of ROS and ATP depletion trigger necrosis in lymphoid cells. Free Radic Biol Med 2008;44:1146-60.

17. Lee SM, Dho SH, Ju SK, Maeng JS, Kim JY, Kwon KS. Cytosolic malate dehydrogenase regulates senescence in human fibroblasts. Biogerontology 2012;13:525-36.

18. Woo HA, Bae SH, Park S, Rhee SG. Sestrin 2 is not a reductase for cysteine sulfinic acid of peroxiredoxins. Antioxid Redox Signal 2009; 11:739-45.

19. Wempe F, De-Zolt S, Koli K, Bangsow T, Parajuli N, Dumitrascu $\mathrm{R}$, et al. Inactivation of sestrin 2 induces TGF-beta signaling and partially rescues pulmonary emphysema in a mouse model of COPD. Dis Model Mech 2010;3:246-53.

20. Lener B, Koziel R, Pircher H, Hutter E, Greussing R, Herndler-Brandstetter $\mathrm{D}$, et al. The NADPH oxidase Nox4 restricts the replicative lifespan of human endothelial cells. Biochem J 2009;423:363-74

21. Rodier F, Campisi J. Four faces of cellular senescence. J Cell Biol 2011;192:547-56.

22. Hwang ES, Hwang SY. Cellular NAD+ level: a key determinant of mitochondrial quality and health. Ann Geriatr Med Res 2017;21(4):149-57.

23. Wang W, Yang X, Lopez de Silanes I, Carling D, Gorospe M. Increased AMP:ATP ratio and AMP-activated protein kinase activity during cellular senescence linked to reduced HuR function. J Biol Chem 2003;278:27016-23.

24. Irrcher I, Ljubicic V, Hood DA. Interactions between ROS and AMP kinase activity in the regulation of PGC-1alpha transcription in skeletal muscle cells. Am J Physiol Cell Physiol 2009;296: C116-23.

25. Jones RG, Plas DR, Kubek S, Buzzai M, Mu J, Xu Y, et al. AMP-activated protein kinase induces a p53-dependent metabolic checkpoint. Mol Cell 2005;18:283-93.

26. Wang W, Yang X, Cristofalo VJ, Holbrook NJ, Gorospe M. Loss of $\mathrm{HuR}$ is linked to reduced expression of proliferative genes during replicative senescence. Mol Cell Biol 2001;21:5889-98.

27. Hay N, Sonenberg N. Upstream and downstream of mTOR. Genes Dev 2004;18:1926-45.

28. Inoki K, Zhu T, Guan KL. TSC2 mediates cellular energy response to control cell growth and survival. Cell 2003;115:57790.

29. Cheng SW, Fryer LG, Carling D, Shepherd PR. Thr2446 is a novel mammalian target of rapamycin (mTOR) phosphorylation site regulated by nutrient status. J Biol Chem 2004;279: 15719-22.

30. Zu Y, Liu L, Lee MY, Xu C, Liang Y, Man RY, et al. SIRT1 promotes proliferation and prevents senescence through targeting LKB1 in primary porcine aortic endothelial cells. Circ Res 2010; 106:1384-93. 